\title{
Biochemical evaluation of phenolic compounds and steviol glycoside from Stevia rebaudiana extracts associated with in vitro antidiabetic potential
}

\begin{abstract}
Apart from being well known to the world as herb-based sweetening additive, Stevia rebaudiana (S. rebaudiana) and its phenolic compounds are considered as a natural antidiabetic alternative to replace synthetic drugs that possess numbers of side effects. Therefore, this study was aimed to evaluate the solvent effects on the extraction of phenolic compounds and steviol glycoside identification associated with antidiabetic potential of the extracts. Total phenolic (TPC) and flavonoid (TFC) content of the extracts were quantified, while the antidiabetic activity of the extracts was determined by $\alpha$-amylase and $\alpha$-glucosidase inhibitory assay. As a matter of interest, TFC was found to be present at the highest concentration in ethanol extract (10.91 mg QE/g), while the presence of TPC showed no significant difference between water extract $(6.65 \mathrm{mg} \mathrm{GAE} / \mathrm{g})$ and other organic solvents. HPLC analysis showed the abundant presence of steviol glycoside in the water extract, the principal compound suggested for treating diabetes. Furthermore, GC-MS analysis has shown the major compounds of 1-hepta-triacotanol, duvatrienediol, dihydroxanthin, $\beta$-amyrin, lupenone, phytol, $\gamma$-sitosterol, agatholic acid and fatty acids were present. In relation to the antidiabetic potential, the effects of the extracts in inhibiting $\alpha$-amylase and $\alpha$-glucosidase activity were investigated in vitro. Interestingly, among allS. rebaudiana extracts, water extract exhibited the most significant $\alpha$-amylase inhibitory activity with IC50 $=8.63 \mu \mathrm{g} / \mathrm{ml}$, comparable to the synthetic drug, acarbose IC50 $=13.73 \mu \mathrm{g} / \mathrm{ml}$. These findings demonstrated that phenolic recovery was highly dependent on extraction solvent and the promising water extract as the best $\alpha$-amylase inhibitory potential with greatest steviol glycoside recovery.
\end{abstract}

Keyword: Stevia rebaudiana; Phenolic content; Steviol glycoside; $\alpha$-Amylase inhibitory activity; $\alpha$-Glucosidase inhibitory activity; Antidiabetic potential 